\title{
Erratum to: Immune maintenance in glaucoma: boosting the body's own neuroprotective potential
}

\author{
Michal Schwartz • Anat London
}

Published online: 23 September 2009

(C) The Author(s) 2009. This article is published with open access at Springerlink.com

\section{Erratum to: j ocul biol dis inform} DOI 10.1007/s12177-009-9025-7

The following article was mistakenly run in volume 2 , issue 2 (June 2009) instead of in the present special issue, for which it was intended. The publisher regrets the error.

\begin{abstract}
Glaucoma, a slow progressive neurodegenerative disorder associated with death of retinal ganglion cells and degeneration of their connected optic nerve fibers, has been classically linked to high intraocular pressure. Regardless of the primary risk factor, degeneration may continue, resulting in further loss of neurons and subsequent glaucomatous damage. During the past decade, scientists and clinicians began to accept that, in addition or as an alternative to fighting off the primary risk factor(s), there is a need to protect the tissue from the ongoing spread of damage - an approach collectively termed "neuroprotection." We found that the immune system, the body's own defense mechanism, plays a key role in the ability of the optic nerve and the retina to withstand glaucomatous conditions. This defense involves recruitment of both innate and adaptive immune cells that together create a protective niche and thereby halt disease progression. The spontaneous immune response might not be sufficient, and therefore, we suggest boosting it by immunization (with the appropriate antigen, at specific timing and predetermined optimal dosing) which may be developed into a suitable therapeutic vaccination to
\end{abstract}

The online version of the original article can be found at http://dx.doi. org/10.1007/s12177-009-9025-7.

M. Schwartz $(\bowtie) \cdot$ A. London

Department of Neurobiology, The Weizmann Institute of Science, Rehovot, Israel

e-mail: michal.schwartz@weizmann.ac.il treat glaucoma. This view of immune system involvement in glaucoma will raise new challenges in glaucoma research, changing the way in which clinicians perceive the disease and the approach to therapy.

Keywords Glaucoma - Neuroprotection .

"Protective autoimmunity". Therapeutic vaccination .

Neurodegenerative diseases · Macrophages

Glaucoma is a major cause of blindness. It represents a significant increasing public health problem, which affects more than 67 million people, worldwide.

Initially described by Hippocrates as a cloudy blue (seacolored) pupil, glaucoma was accepted in the middle of the nineteenth century as a disease resulting from elevated intraocular pressure (IOP). Ever since, large, multicenter clinical trials and several laboratory studies recognized ocular hypertension as the most important factor in the development of glaucoma. Consequently, IOP was a major target for treating glaucoma, and thus, all the currently available glaucoma therapies involve medical or surgical procedures designed to lower IOP [1-5]. Although significantly successful in attenuation of progressive visual loss among the treated patients $[1-3,6]$, it appeared that reducing IOP did not fully arrest the disease in all cases. Some patients continued to display glaucomatous damage even after their IOP levels were controlled [7-9], while others developed retinal degeneration in the absence of any increase in IOP, a variant of the disease termed normal tension glaucoma (NTG). It was thus realized that there is more to glaucoma than just increased IOP.

Glaucoma is a complex multivariate disease, initiated by several risk factors (with elevated IOP as only one of them). Following the initial damage, the degenerating neurons 
create a hostile milieu, characterized by oxidative stress and free radicals [10-12], excessive amounts of glutamate and excitotoxicity [13, 14], increased calcium concentration, deprivation of neurotrophins and growth factors, abnormal accumulation of proteins, and apoptotic signals [15-20]. These factors contribute to the secondary damage, resulting in further neuronal loss. The secondary damage ensuing from this noxious environment is shared by many neurodegenerative diseases including Alzheimer's disease, Parkinson's disease, and amyotrophic lateral sclerosis [21]. This observation, together with other similarities between glaucoma and common neurodegenerative disorders, has led to the current view of glaucoma as a neurodegenerative disease. Thus, major research efforts are currently directed at attempts to prevent disease progression and the secondary spread of damage by a neuroprotective approach. Regardless of the primary risk factor, glaucoma treatment is now focused on the degenerating neurons, rather than on other potential etiologies. Thus, the field of glaucoma research is now moving towards neuroprotection.

Despite the massive efforts, the clinical use of neuroprotective agents in glaucoma is not yet a reality. Many agents have been investigated for neuroprotective properties, including free radical scavengers, antiexcitotoxic agents, inhibitors of apoptosis, anti-inflammatory agents, neurotrophic factors, metal ion chelators, ion channel modulators, and gene therapy. However, none of these agents has been approved to date. Part of the delay in bringing a neuroprotectant drug to clinical practice is due to the attempt to deal with one single risk factor at a time. This challenge is doomed to fail, as each of the risk factors contributes to the neuronal spread of damage at different overlapping stages of disease progression, and thus it is almost impossible to hit the right target at the correct time. Instead of focusing individually on a single risk factor, our group has demonstrated during the past decade that harnessing the immune system, the body's own defense mechanism, can contribute to minimizing the spread of damage that derives from the multiple risk factors of glaucoma, and in this way, the properly regulated immune response can restore central nervous system (CNS) homeostasis.

Any CNS insult, whether it is acute or chronic, involves the activation of immune cells. Immune activation in the "immune privileged" CNS has long been perceived as a key factor contributing to the pathology and leading to the further spread of damage and neuronal death. As a result, research efforts were directed at attempts to diminish this apparent obstacle to recovery by the use of steroids and anti-inflammatory drugs. Although this approach was often associated with some transient improvement, several of these anti-inflammatory agents have not only fallen short in resolving CNS neurodegenerative disorder, but were rather detrimental [22]. These failures are not surprising and are consistent with our results, demonstrating a protective role of the regulated immune response following CNS insult. Thus, it might not be helpful to globally diminish the immune response and prevent the natural healing of the brain. When mice are challenged with neurodegenerative conditions or neuronal injury, such as those existing in experimental glaucoma models (optic nerve crush, induced glutamate toxicity, or increased IOP), reduced neuronal survival is detected $[23,24]$ in immune deficient mice, such as nude mice, or thymectomized animals. A similar phenomenon is observed following administration of steroids [25]. When the relevant immune cells/activity is restored, the ability of the retina to cope with the damage returns, further demonstrating the need for a spontaneous physiological immune response that can protect the neurons from the glaucomatous damage.

Following the discovery of the essential role of the immune system in protecting the retinal neuronal cells, we wished to understand which immune cells are involved in this process. The immune system is mainly associated with the recognition of pathogens. Any immune response to "self-antigens" was traditionally considered detrimental and was reflexively seen as an autoimmune disease. However, in the case of damage to the CNS in general and to the retinal ganglion cells (RGC) in particular, we have learned that $\mathrm{T}$ cells specific for certain CNS-specific self-antigens can protect the injured neurons from death; passive transfer of $\mathrm{T}$ cells specific to myelin basic protein (MBP) reduces the loss of RGC after traumatic optic nerve injury [26]. This concept of defensive immune response directed towards specific self-antigens, residing in the site of damage, was established by our group and was termed "protective autoimmunity" [23].

Unfortunately, the spontaneous autoimmune response is probably insufficient or insufficiently regulated to promote significant CNS protection. This can account for most of the failures of spontaneous recovery following acute and chronic CNS insults. We, therefore, suggested boosting this protective autoimmune response by either passive transfer of self-reactive $\mathrm{T}$ cells or active immunization using selfantigens [23, 26-31]. With either approach, the choice of the antigen is critical; only self-antigens that are present at the damage site will promote neuroprotection. Thus, in our experiments, only the transfer of $\mathrm{T}$ cells reactive to myelin components that are expressed at the damage site and not to the irrelevant antigen, OVA, enhanced the survival levels of RGC [29]. A similar phenomenon is evident in active immunization; only the use of whole retinal homogenate, rather than spinal cord homogenate, is effective in protecting the degenerating retina [29]. Notably, these T cells are also effective when directed to cryptic epitopes of MBP, as well as toward other myelin-derived altered peptides [32, 33]. In this way, we can offer neuroprotection without the risk of 
causing an autoimmune disease. One such excellent example is the use of Copaxone (GA), a drug approved by the Food and Drug Administration for treating multiple sclerosis; this agent consists of a synthetic polymer that weakly stimulates a wide range of self-reactive $T$ cells. Vaccination with GA significantly reduces RGC loss, by controlling the milieu of the nerve and retina, making it less hostile to neuronal survival and allowing RGC to better withstand the stress [27, 34-37]. Thus, Copaxone provides ideal protection without the risk of promoting an autoimmune disease.

Once activated by self-reactive antigens, the protective autoimmune $\mathrm{T}$ cells create a neuroprotective environment that prevents/attenuates the secondary spread of damage by changing the nature of the local innate immune response (see Fig. 1). T cells provide or facilitate recruitment of blood monocytes, which, upon arrival to the damaged area, contribute to restoration of homeostasis by producing immunoregulatory cytokines, and thereby allow production of the growth factors, chemokines, and cytokines that properly activate resident microglia. Such activated microglia/macrophages can take up glutamate, remove debris, and produce growth factors. In addition, instead of contributing to the destructive ongoing inflammation, these cells do not produce agents that are part of their cytotoxic mechanism, such as TNF- $\alpha$, which the eye, like the brain, tolerates poorly [38-42]; rather, these "alternatively" activated microglia/macrophages participate in the regulation of the local inflammation and restoration of homeostasis [43]. During the course of our studies, we have learned that both innate and adaptive immune cells, when properly regulated, can promote neuroprotection in the degenerating RGC. Interestingly, the same $\mathrm{T}$ cells that can lead to the development of an autoimmune disease can protect neurons under pathological conditions. This sug-
Fig. 1 Therapeutic vaccination for glaucoma: immune modulation to cease spread of damage. Following the initial insult, dying neurons create a hostile environment that includes apoptotic signals, free radicals and oxidative stress, toxic levels of glutamate, ionic imbalance, abnormal protein behavior, and deprivation of growth factors and neurotrophins. These factors and others, at different overlapping stages of disease progression, mediate further spread of damage and secondary degeneration, leading to the loss of neighboring, still healthy neurons. As a therapeutic approach, we suggest using an active or passive therapeutic vaccination that will lead to a regulated autoimmune response. In this way, autoimmune $\mathrm{T}$ cells, recognizing specific CNS antigens residing at the damaged retina, will participate in the recruitment and activation of macrophages, leading to the regulation of the local inflammation and restoration of homeostasis

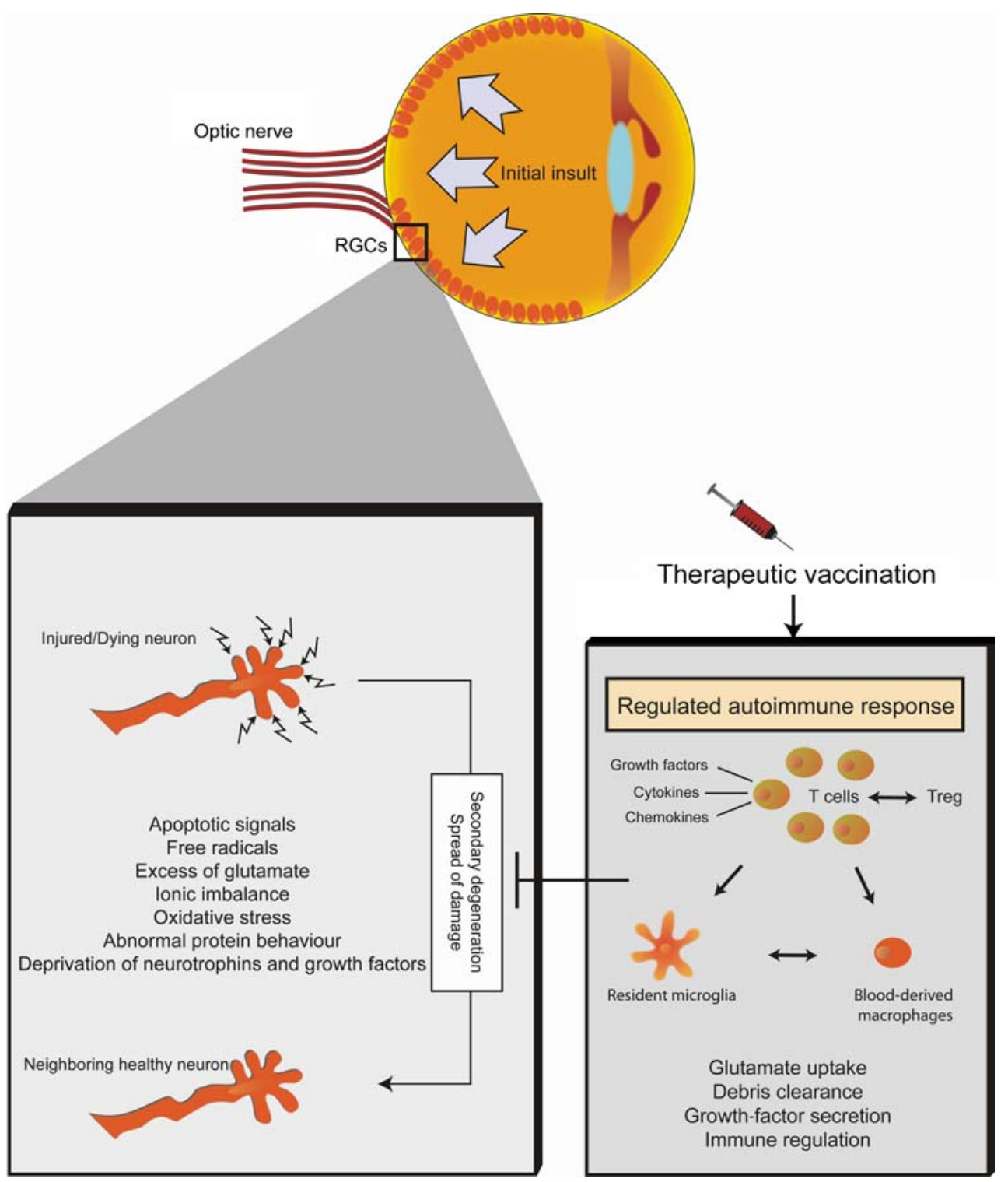


gests the obvious question: how is the protective response by autoimmune $\mathrm{T}$ cells enabled without the potential risk of creating an autoimmune disease? We found that regulation is an important factor in this equation involving protective autoimmunity on one hand and autoimmune disease on the other. Self-reactive T cells are constitutively controlled by a subpopulation of regulatory $\mathrm{T}$ cells that are part of the physiological immune network, maintaining the autoimmune response in a form that protects the eye rather than hurting it. These $\mathrm{T}$ regulatory cells are themselves amenable to regulation and control $[44,45]$.

Other than their role in CNS protection, immune cells are also involved in cell renewal. Many factors affect adult neurogenesis - the creation of new neurons. We suggest that immune components are also involved in this process, as they transmit a message from the local niche to the neural progenitor cells (NPC). We have previously shown that immune cells, recognizing self-CNS antigens, not only promote protection, but also contribute to the maintenance of neurogenesis in the adult hippocampus [46]. More recently, we showed that not only immune cells, but also immune receptors, are involved in the neurogenic process [47]. We found that Toll-like receptors (TLR), which are innate receptors recognizing extrinsic and intrinsic components, are expressed on NPC and regulate adult hippocampal neurogenesis. When examining the retina, we found that TLR4 is a key player in regulating neurogenesis: TLR4deficient mice showed increased proliferation and neuronal differentiation. This deficiency is not sufficient to extend the postnatal neurogenesis period, but when combined with growth factors, TLR4 deficiency contributes to the resumption of neurogenesis [48]. The identification of TLR4 as a regulator of neurogenesis may provide a promising therapeutic direction for promoting neurogenesis in the adult eye under pathological conditions.

\section{Concluding remarks}

In the course of our studies, we learned that the immune system is pivotal for CNS maintenance and for withstanding neurodegenerative conditions. Immune deficiency or immune suppression impairs the recovery process, whereas boosting a self-specific immune response, by either passive or active immunization, promotes recovery. In order to optimally induce recovery, the immune response should be a well-regulated autoimmune response in which the choice of antigen, dosing, and regimen are critical. Both innate and adaptive immune responses are involved in the neuroprotective process. In addition to their roles in neuroprotection, immune components are key regulators of neurogenesis and, therefore, can serve as therapeutic targets in the adult eye under pathological conditions.
We suggest recruiting the immune system, which is the body's own defense system, to fight off the various degenerative conditions that evolve in glaucoma. This immune recruitment can be boosted by systemic selfspecific vaccination, involving both innate and adaptive cells, and eventually leading to improved recovery and preservation of the retinal neuronal tissue.

Open Access This article is distributed under the terms of the Creative Commons Attribution Noncommercial License which permits any noncommercial use, distribution, and reproduction in any medium, provided the original author(s) and source are credited.

\section{References}

1. Quigley HA, Maumenee AE. Long-term follow-up of treated open-angle glaucoma. Am J Ophthalmol. 1979;87(4):519-25.

2. Kass MA, et al. The ocular hypertension treatment study: a randomized trial determines that topical ocular hypotensive medication delays or prevents the onset of primary open-angle glaucoma. Arch Ophthalmol. 2002;120(6):701-13. discussion 829-30.

3. Leske MC, et al. Factors for glaucoma progression and the effect of treatment: the early manifest glaucoma trial. Arch Ophthalmol. 2003;121(1):48-56.

4. Johnson EC, et al. The use of cyclodialysis to limit exposure to elevated intraocular pressure in rat glaucoma models. Exp Eye Res. 2006;83(1):51-60.

5. Nickells RW, et al. Surgical lowering of elevated intraocular pressure in monkeys prevents progression of glaucomatous disease. Exp Eye Res. 2007;84(4):729-36.

6. Heijl A, et al. Reduction of intraocular pressure and glaucoma progression: results from the Early Manifest Glaucoma Trial. Arch Ophthalmol. 2002;120(10):1268-79.

7. Jay JL, Allan D. The benefit of early trabeculectomy versus conventional management in primary open angle glaucoma relative to severity of disease. Eye. 1989;3(Pt 5):528-35.

8. Nouri-Mahdavi K, et al. Outcomes of trabeculectomy for primary open-angle glaucoma. Ophthalmology. 1995;102(12):1760-9.

9. Brubaker RF. Delayed functional loss in glaucoma. LII Edward Jackson Memorial Lecture. Am J Ophthalmol. 1996;121(5):47383.

10. Oku H, et al. Retinal toxicity of nitric oxide released by administration of a nitric oxide donor in the albino rabbit. Invest Ophthalmol Vis Sci. 1997;38(12):2540-4.

11. Levkovitch-Verbin H, et al. RGC death in mice after optic nerve crush injury: oxidative stress and neuroprotection. Invest Ophthalmol Vis Sci. 2000;41(13):4169-74.

12. Tezel G. Oxidative stress in glaucomatous neurodegeneration: mechanisms and consequences. Prog Retin Eye Res. 2006;25 (5):490-513.

13. Olney JW. Glutaate-induced retinal degeneration in neonatal mice. Electron microscopy of the acutely evolving lesion. J Neuropathol Exp Neurol. 1969;28(3):455-74.

14. Olney JW, et al. The role of specific ions in glutamate neurotoxicity. Neurosci Lett. 1986;65(1):65-71.

15. McKinnon SJ. Glaucoma, apoptosis, and neuroprotection. Curr Opin Ophthalmol. 1997;8(2):28-37.

16. Oltvai ZN, Korsmeyer SJ. Checkpoints of dueling dimers foil death wishes. Cell. 1994;79(2):189-92.

17. Levin LA, et al. Identification of the bcl-2 family of genes in the rat retina. Invest Ophthalmol Vis Sci. 1997;38(12):2545-53. 
18. Thornberry NA. Caspases: key mediators of apoptosis. Chem Biol. 1998;5(5):R97-103.

19. Thornberry NA, Lazebnik Y. Caspases: enemies within. Science. 1998;281(5381):1312-6.

20. Quigley HA. Neuronal death in glaucoma. Prog Retin Eye Res. 1999;18(1):39-57.

21. Schwartz M. Lessons for glaucoma from other neurodegenerative diseases: can one treatment suit them all? J Glaucoma. 2005;14 (4):321-3

22. Masters C, Wyss-Coray T, Pasinetti GM. Anti-inflammatory drugs fall short in Alzheimer's disease. Nat Med, 2008;14:916.

23. Yoles E, et al. Protective autoimmunity is a physiological response to CNS trauma. J Neurosci. 2001;21(11):3740-8.

24. Kipnis J, et al. Neuronal survival after CNS insult is determined by a genetically encoded autoimmune response. J Neurosci. 2001;21(13):4564-71.

25. Bakalash S, et al. Antigenic specificity of immunoprotective therapeutic vaccination for glaucoma. Invest Ophthalmol Vis Sci. 2003;44:3374-81.

26. Moalem G, et al. Autoimmune $\mathrm{T}$ cells protect neurons from secondary degeneration after central nervous system axotomy. Nat Med. 1999;5(1):49-55.

27. Schori $\mathrm{H}$, et al. Vaccination for protection of retinal ganglion cells against death from glutamate cytotoxicity and ocular hypertension: implications for glaucoma. Proc Natl Acad Sci U S A. 2001;98(6):3398-403.

28. Schori H, Yoles E, Schwartz M. T-cell-based immunity counteracts the potential toxicity of glutamate in the central nervous system. J Neuroimmunol. 2001;119(2):199-204.

29. Avidan $\mathrm{H}$, et al. Vaccination with autoantigen protects against aggregated beta-amyloid and glutamate toxicity by controlling microglia: effect of $\mathrm{CD}^{+}{ }^{+} \mathrm{CD} 25^{+}$T cells. Eur J Immunol. 2004;34 (12):3434-45.

30. Hauben E, et al. Passive or active immunization with myelin basic protein promotes recovery from spinal cord contusion. J Neurosci. 2000;20(17):6421-30.

31. Hauben E, Schwartz M. Therapeutic vaccination for spinal cord injury: helping the body to cure itself. Trends Pharmacol Sci. 2003;24:7-12.

32. Fisher $\mathrm{J}$, et al. Vaccination for neuroprotection in the mouse optic nerve: implications for optic neuropathies. J Neurosci. 2001;21 (1):136-42.

33. Mizrahi T, Hauben E, Schwartz M. The tissue-specific selfpathogen is the protective self-antigen: the case of uveitis. J Immunol. 2002;169:5971-7.
34. Kipnis J, et al. T cell immunity to copolymer 1 confers neuroprotection on the damaged optic nerve: possible therapy for optic neuropathies. Proc Natl Acad Sci U S A. 2000;97(13):7446-51.

35. Kipnis J, Schwartz M. Dual action of glatiramer acetate (Cop-1) in the treatment of CNS autoimmune and neurodegenerative disorders. Trends Mol Med. 2002;8:319-23.

36. Benner EJ, et al. Therapeutic immunization protects dopaminergic neurons in a mouse model of Parkinson's disease. Proc Natl Acad Sci U S A. 2004;101(25):9435-40.

37. Schwartz M. Modulating the immune system: a vaccine for glaucoma? Can J Ophthalmol. 2007;42(3):439-41.

38. Moalem G, et al. Autoimmune T cells retard the loss of function in injured rat optic nerves. J Neuroimmunol. 2000;106(1-2):189-97.

39. Butovsky O, Hauben E, Schwartz M. Morphological aspects of spinal cord autoimmune neuroprotection: colocalization of T cells with B7-2 (CD86) and prevention of cyst formation. FASEB J. 2001;15(6):1065-7.

40. Barouch R, Schwartz M. Autoreactive T cells induce neurotrophin production by immune and neural cells in injured rat optic nerve: implications for protective autoimmunity. FASEB J. 2002;16: 1304-6.

41. Butovsky $\mathrm{O}$, et al. Activation of microglia by aggregated betaamyloid or lipopolysaccharide impairs MHC-II expression and renders them cytotoxic whereas IFN-gamma and IL-4 render them protective. Mol Cell Neurosci. 2005;29(3):381-93.

42. Shaked I, et al. Protective autoimmunity: interferon-gamma enables microglia to remove glutamate without evoking inflammatory mediators. J Neurochem. 2005;92:997-1009.

43. Shechter R, et al. Infiltrating blood-derived macrophages: vital immunoregulatory cells in spinal cord repair. Plos Med. 2009 (in press)

44. Kipnis J, et al. Neuroprotective autoimmunity: naturally occurring $\mathrm{CD} 4{ }^{+} \mathrm{CD} 25^{+}$regulatory $\mathrm{T}$ cells suppress the ability to withstand injury to the central nervous system. Proc Natl Acad Sci U S A. 2002;99:15620-5.

45. Kipnis J, et al. Dopamine, through the extracellular signalregulated kinase pathway, downregulates $\mathrm{CD} 4{ }^{+} \mathrm{CD} 25^{+}$regulatory T-cell activity: implications for neurodegeneration. J Neurosci. 2004;24:6133-43.

46. $\mathrm{Ziv} \mathrm{Y}$, et al. Immune cells contribute to the maintenance of neurogenesis and spatial learning abilities in adulthood. Nat Neurosci. 2006;9(2):268-75.

47. Rolls A, et al. Toll-like receptors modulate adult hippocampal neurogenesis. Nat Cell Biol. 2007;9(9):1081-8.

48. Shechter R, et al. Toll-like receptor 4 restricts retinal progenitor cell proliferation. J Cell Biol. 2008;183(3):393-400. 